\title{
Realization of Fractional Order Controllers by Using Multiple Tuning-Rules
}

\author{
Zhe Yan, Jing He, Yingyan Li, Kai Li and Changqi Song \\ Department of Automation, Harbin University of Science and Technology
}

yanzhehrb@163.com

\begin{abstract}
Fractional order PID controller has two degrees of freedom more than the integer order PID controller, and it has better control performance. The paper briefly describes the fractional order PID controller, and through Ziegler-Nichols type tuning rules, Padula \& Visioli tuning rules and tuning by minimization methods to achieve fractional order PID controllers respectively. Additionally, the Oustaloup approximation methods are both introduced. Based on these methods, we have considered the steam pressure model and the vehicle wire control system. The simulation result shows that control system stability can be obtained by using the tuning-rules proposed in this paper.
\end{abstract}

Keywords: fractional order calculus; $P I^{\lambda} D^{\mu}$ controller; tuning rules

\section{Introduction}

Nowadays, the better understanding of the potential of fractional calculus and the increasing number of studies related to the applications of fractional order controllers in many areas of science and engineering have led to the importance of studying aspects such as the analysis, design, tuning and implementation of these controllers. Fractional order calculus is an area of mathematics that deals with derivatives and integrals from non-integer orders. In other tools, it is a generalization of the traditional calculus that leads to similar concepts and tools, but with a much wider applicability.

In theory, the control systems can include both the fractional order dynamic system or plant to be controlled and the fractional-order controller. However, in control practice, more common is to consider the fractional order controller. This is due to the fact that the plant model may have already been obtained as an integer order model in classical sense. In most cases, our objective is to apply the fractional order control (FOC) to enhance the system control performance.

In this paper, basic ideas of fractional order PID controllers are presented first. Additionally, design of FOPID controllers is presented via different approaches including Ziegler-Nichols tuning rules, and the Padula \& Visioli method. According to the characteristic of main steam controlled object such as large inertia and delay, a fractional PID controller is designed by using the Ziegler-Nichols type tuning rules. Rely on the Oustaloup approximation, we have considered Vehicle wire control system. Finally, conclusions are drawn in part 4.

\section{Basic Ideas of Fractional Order PID Controller}

According to a survey of the state of process control systems in 1989 conducted by the Japan Electric Measuring Instrument Manufacturer's Association, more than 90 percent of the 
control loops were of the PID type. It was also indicated in that a typical paper mill in Canada has more than 2,000 control loops and that 97 percent use PI control. Therefore, the industrialist had concentrated on PI/PID controllers and had already developed on-button type relay auto-tuning techniques for fast, reliable PI/PID control yet with satisfactory performance [1-4].

The most common form of a fractional order PID controller is the $\mathrm{PI}^{\lambda} \mathrm{D}^{\mu}$ controller [4], involving an integrator of order $\lambda$ and a differentiator of order $\mu$ where $\lambda$ and $\mu$ can be any real numbers. The transfer function of such a controller has the following form

$$
\begin{gathered}
G_{C}(\mathrm{~s})=\frac{U(s)}{E(s)}=k_{P}+k_{I} \frac{1}{s^{\lambda}}+k_{D} s^{\mu} \\
(\lambda, \mu>0)
\end{gathered}
$$

where the $\mathrm{E}(\mathrm{s})$ is an error, and $\mathrm{U}(\mathrm{s})$ is controller's output. The control signal $\mathrm{u}(\mathrm{t})$ can then be expressed in the time domain as

$$
u(t)=k_{P} e(t)+k_{I} D^{-\lambda} e(t)+k_{D} D^{\mu} e(t)
$$

Clearly, selecting $\lambda=1$ and $\mu=1$, a classical PID controller can be recovered. Using $\lambda=1$, $\mu=0$, and $\lambda=0, \mu=1$, respectively, corresponds to the conventional PI \& PD controllers. All these classical types of PID controllers are special cases of the $\mathrm{PI}^{\lambda} \mathrm{D}^{\mu}$ controller given by (1). It can be expected that the $\mathrm{PI}^{\lambda} \mathrm{D}^{\mu}$ controller may enhance the systems control performance. One of the most important advantages of the $\mathrm{PI}^{\lambda} \mathrm{D}^{\mu}$ controller is the possible better control of fractional order dynamical systems. Another advantage lies in the fact that the $\operatorname{PI}^{\lambda} \mathrm{D}^{\mu}$ controllers are less sensitive to changes of parameters of a controlled system [4]. This is due to the two extra degrees of freedom to better adjust the dynamical properties of a fractional order control system. It is pointed out in [5] that a band-limit implementation of fractional order controller is important in practice, and the finite dimensional approximation of the fractional order controller should be done in a proper range of frequencies of practical interest.

\section{The Tuning Rules of Fractional-order $\operatorname{PI}^{\lambda} D^{\mu}$ Controller}

\subsection{A Fractional Order PID Controller Design Method-- Tuning by minimization}

Tuning by minimization is a tuning method for fractional PIDs [6], in with we begin by devising a desirable behavior for our controller system, described by five specifications:

1) The gain-crossover frequency $\omega_{\text {cg }}$ is to have some specified value :

$$
\left|C\left(j \omega_{c g}\right) G\left(j \omega_{c g}\right)\right|=0 d B
$$

2) The phase margin $\phi_{m}$ is have some specified value:

$$
-\pi+\phi_{m}=\arg \left[C\left(j \omega_{c g}\right) G\left(j \omega_{c g}\right)\right\rfloor
$$

3) To reject high-frequency noise, the closed loop transfer function must have a small magnitude at high frequencies $\omega_{\mathrm{h}}$, its magnitude is to be less than some specified gain $\mathrm{H}$ : 


$$
\left|\frac{C\left(j \omega_{h}\right) G\left(j \omega_{h}\right)}{1+C\left(j \omega_{h}\right) G\left(j \omega_{h}\right)}\right|<H
$$

4) To reject output disturbances and closely follow references, the sensitivity function must have a small magnitude at low frequencies; hence, at some specified frequency $\omega_{1}$, its magnitude is to be less than some specified gain $\mathrm{L}$ :

$$
\left|\frac{1}{1+C\left(j \omega_{l}\right) G\left(j \omega_{l}\right)}\right|<L
$$

5) To be robust when gain variations of the plant occur, the phase of the open-loop transfer function is to be (at least roughly) constant around the gain-crossover frequency:

$$
\frac{d}{d \omega} \arg [C(j \omega) G(j \omega)]_{\omega=\omega_{c g}}=0
$$

Then the five parameters of the fractional PID are to be found using the Nelder-Mead direct search simplex minimization method. This derivative-free method is used to minimize the difference between the desired performance achieved by the controller.

\subsection{Example}

The optimal FOPD model (fit it with a fixed model structure known as first-order plus delay) is given as follows:

$$
G(s)=\frac{3.13}{433.53 \mathrm{~s}+1} \mathrm{e}^{-50 s}
$$

Now, let us consider the $\mathrm{PI}^{\lambda} \mathrm{D}^{\mu}$ controller. Here, the design specifications required for the system are: $\phi_{m}=60^{\circ}, \omega_{c \bar{c}} 0.008 \mathrm{rad} / \mathrm{s}$.

Selecting $\lambda=0.8968$ and $\mu=0.4773$, the fractional order PID controller can be designed by the proposed method in this paper. Thus, using our tuning algorithm in the paper, one can have $\mathrm{Kp}=0.6152, \mathrm{Ki}=0.0100$, and $\mathrm{Kd}=4.3867$. The fractional order PID controller is then designed as

$$
G_{C}(s)=0.6152+\frac{0.0100}{s^{0.8968}}+4.3867 s^{0.4773}
$$

The step response of the closed-loop system is illustrated in Figure 1. The Bode plots of the open-loop system $C(s) \cdot G(s)$ are shown in Figure 2. As can be observed, specifications of gain crossover frequency and phase margin are met. Besides, the phase of the system is forced to be flat at $\omega_{c g}$ and hence to be almost constant within an interval around $\omega_{c g}$. 


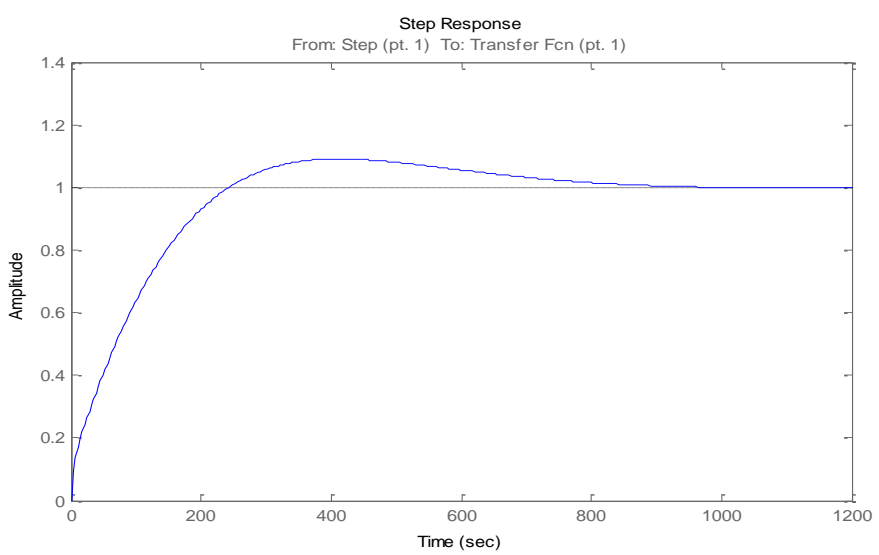

Figure 1. Step Response of the Closed-loop

The fractional PID tuning by minimisation have five conditions. To satisfy them all numerical optimisation algorithms may be used. This is effective, howere allows local minima to be obtained. In practice most solutions found with this optimisation method are well enough, but they strongly depend on initial estimates of the parameters provided. Some may be discarded because they are unfeasible or lead to unstable loops, but in many cases it is possible to find more than one acceptable fractional PID; in others, only well-chosen initial estimates of the parameters allow finding a solution.

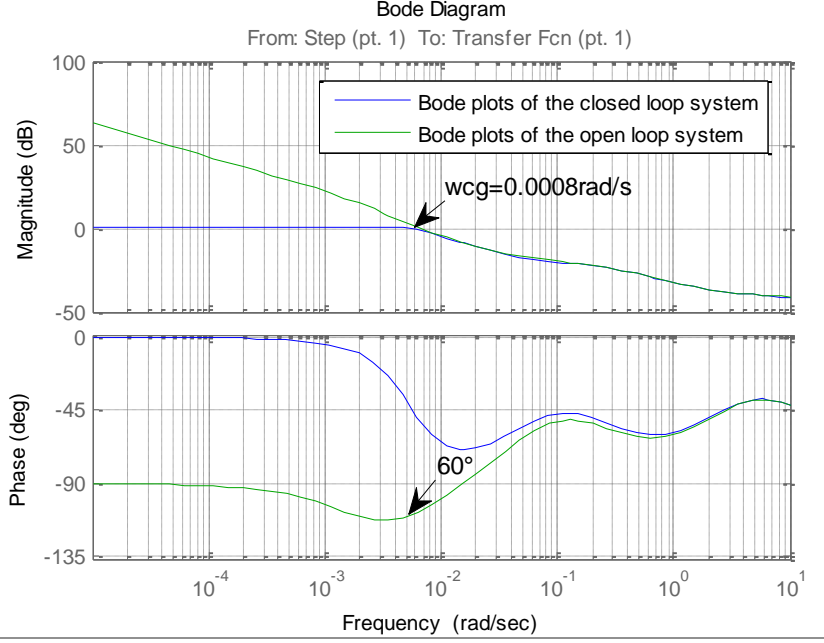

Figure 2. Bode Plot of FOPID Controller Designed

\subsection{Vehicle wire control system with the Oustaloup approximation}

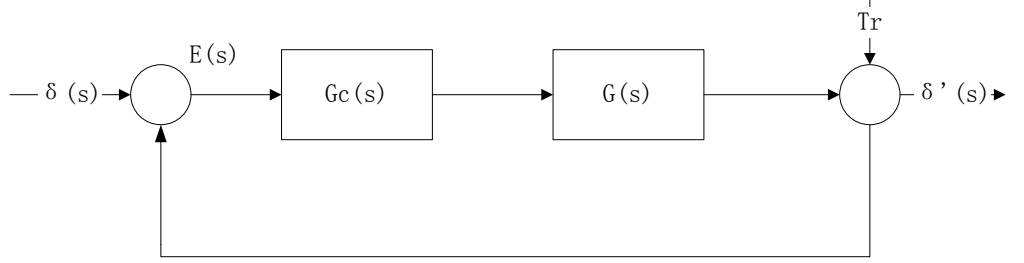

Figure 3.System Structure with Fractional $\mathbf{P I}^{\lambda} \mathbf{D}^{\mu}$ Controller 
For fractional order simulation, usually use the approximation of high order system to replace, Oustaloup approximation algorithm is the most commonly used method. Based on the approximation algorithm, fractional-order of higher order integral system can be directly calculated. Because of the limitations of Oustaloup algorithm itself, $\omega_{\mathrm{a}} \omega_{b}=1$ should be chosen.

The simulation system structure diagram was shown in Figure 3. The plant considered was

$$
G(s)=\frac{1}{0.0042 s^{3}+0.48 s^{2}+1.03 s}
$$

Parameters of controller used in the design process are as follows: $\omega_{c g}=0.99 \mathrm{rad} / \mathrm{s}$ $\omega_{l}=0.001 \mathrm{rad} / \mathrm{s}, \omega_{h}=100 \mathrm{rad} / \mathrm{s}, \phi m=45.9^{\circ}, H=-10 \mathrm{~dB}, N=-20 \mathrm{~dB}$. We can obtain the transfer function of controller

$$
G_{c f}(s)=0.182+\frac{0.7973}{s^{-0.6029}}+0.4994 s^{0.3858}
$$

The open-loop Bode diagram and the closed-loop Bode diagram are shown in the Figure 4 and Figure 5.Using fractional order control, System response time and overshoot volume are almost unchanged. Therefore adopt the $\mathrm{PI}^{\lambda} \mathrm{D}^{\mu}$ fractional order control system that can track signal more accurately and the effect is more ideal.

The Oustaloup approximation has the superiority. However the quality of the this approximation method may not be satisfied in high and low frequency bands near the fitting frequency bounds.

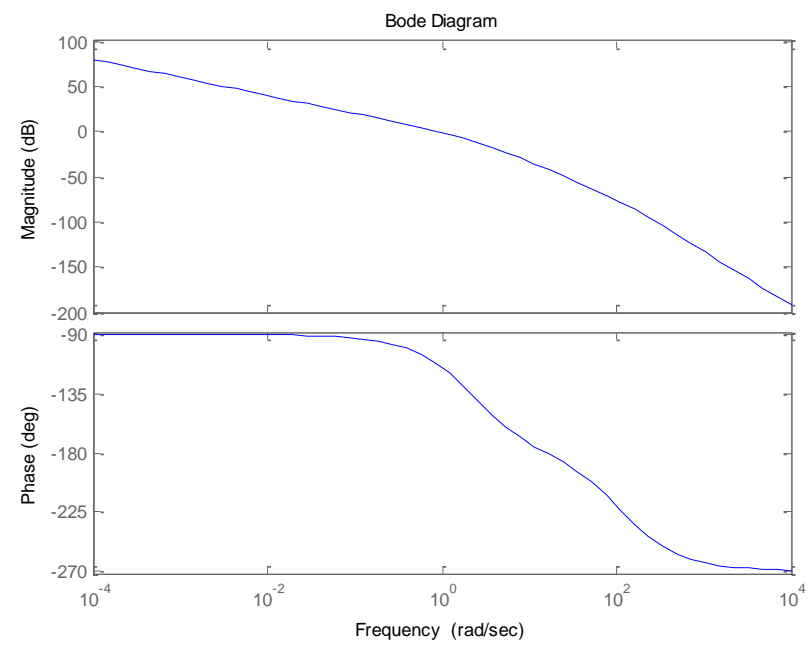

Figure 4. Bode of Open Loop System 


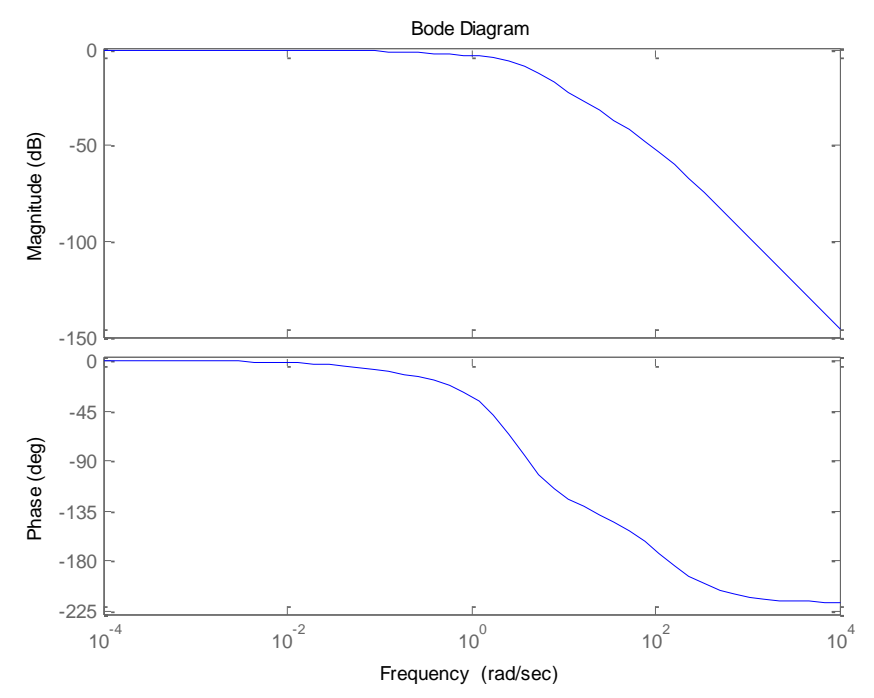

Figure 5. Bode of Closed Loop System

\subsection{Ziegler-Nichols Type Tuning Rules}

In the previous a tuning method for fractional PIDs are presented. This rule is effective but allows local minima to be obtained. Even if a model is known rules quickly provide a starting point for fine tuning. The two methods of the two previous subsections require a model of the plant to control. On the contrary, the tuning rules of the two following subsections try to emulate what happens with existing tuning rules for integer PIDs. They bear similarities to the first rule proposed by Ziegler-Nichols for integer PIDs, making use of the same plant time response data: that rule assumes the plant to have an S-shaped unit-step response. The simplest plant to have S-shaped step response can be described by

$$
G(\mathrm{~s})=\frac{K}{T s+1} e^{-s L}
$$

Valerio and Costa have employed the minimization tuning method to plants given by (12) for several values of $\mathrm{L}$ and $\mathrm{T}$, with $\mathrm{K}=1$. They were designed for the following specifications:

a) Gain crossover frequency $\omega_{c g}=0.5$

b) Phase margin $\phi_{m}=2 / 3 \pi$

c) $\omega_{\mathrm{t}}=10 \mathrm{rad} / \mathrm{sec}, \omega_{\mathrm{s}}=0.01 \mathrm{rad} / \mathrm{sec}$

d) $|S(j \omega)| \leq-20 d B,|T(j \omega)| \leq-10 d B$

\subsection{Example: the steam pressure model}

The steam pressure model can be modeled by the following FOPDT dynamic:

$$
G(s)=\frac{1}{s+1} e^{-0.1 s}
$$




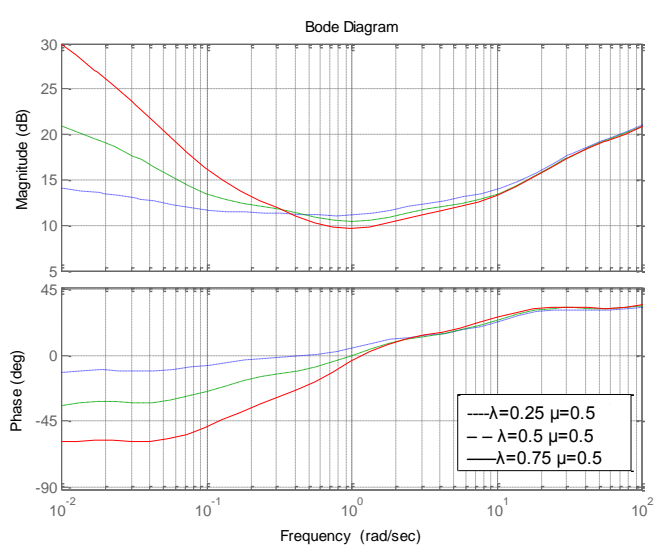

Figure 6.Bode plot of the orders varation-integral order

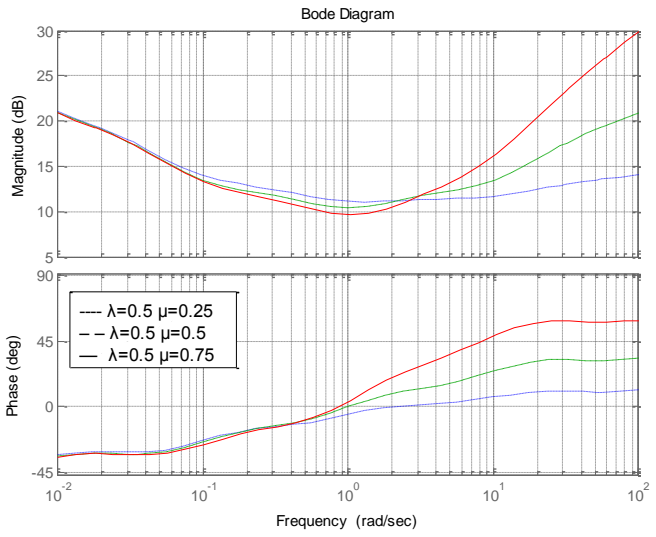

Figure 7.Bode plot of the orders varation-differential order

From the frequency characteristic of fractional order Figure 6, the Bode plot of the fractional order controllers with $\lambda=0.25,0.5,0.75, \mu=0.5 \quad\left(K_{p}=2, K_{i}=1, K_{d}=1\right)$ . The Figure 7 shows the Bode plot of the fractional order controllers with $\mu=0.25,0.5,0.75, \lambda=0.5$

$\left(K_{p}=2, K_{i}=1, K_{d}=1\right)$. In general control system of the low-frequency and the highfrequency, the characteristics of phase frequency and amplitude frequency are affected by $\lambda$ and $\mu$. Fractional order controllers have two more factors than the traditional integer order PID controller, and make the design more flexible, this makes the fractional order controller can better apply in the steam pressure model.

The step response of the process is of S-shaped type and we can use the Ziegler-Nichols type tuning rules for our FOPID controller. Controllers obtained with the first and the second set of tuning rules and with the first Ziegler-Nichols rule are

$$
\left\{\begin{array}{l}
G_{C_{1}}(s)=0.5021+\frac{0.4892}{s^{1.4198}}+0.2045 s^{1.0189} \\
G_{C_{2}}(s)=1.3012+\frac{1.2589}{s^{1.1230}}-0.2601 s^{0.1533} \\
G_{Z N}(s)=12+\frac{60}{s}+0.6 s
\end{array}\right.
$$

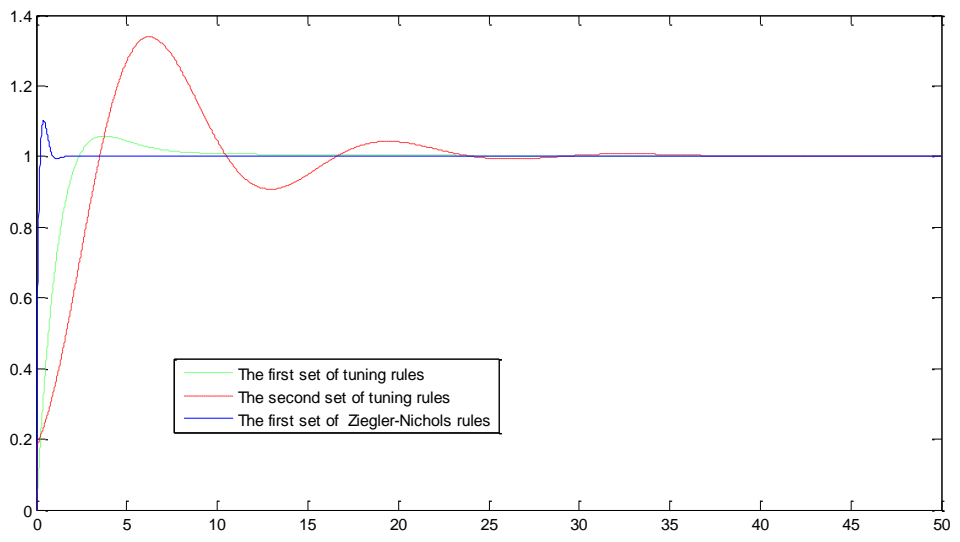

Figure 8.Step response of the steam pressure model with the first set of tuning rules and the second and with the first Ziegler-Nichols rule 
Corresponding step responses are given in Figure 8. It is to be noticed that fractional PIDs can deal with a clearly broader range of values of $\mathrm{K}$. This is likely because the specifications the integer PID tries to achieve are different: that is why responses are all faster, at the cost of greater overshoots. More important is that the overshoot is fairly constant with fractional PIDs, at least for those values closer to 1 . This is because fractional PIDs attempt to verify (11), which the integer PID does not.

\subsection{The Padula \& Visioli Tuning Rules}

A new set of tuning rules are presented for FOPID controllers by Padula \& Visioli [7, 8]. Based on FOPDT models, the tuning rules have been devised in order to minimise the integrated absolute error with a constraint on the maximum sensitivity. We consider the unityfeedback control. In case an unstable process is considered, the process is assumed to have a UFOPDT dynamics as one given by (13).

The process dynamics can be conveniently characterized by the normalized dead time and define

$\mathrm{d}$ as

$$
\tau=\frac{L}{T}
$$

which represents a measure of difficulty in controlling the process.

The FOPID controller is defined as

$$
C(s)=K_{p} \frac{T_{i} s^{\lambda}+1}{T_{i} s^{\lambda}} \frac{T_{d} s^{\mu}+1}{T_{f} s^{\mu}+1}
$$

where $\mathrm{Kp}$ is the proportional gain, $\mathrm{Ti}$ is the integral time constant, $\mathrm{Td}$ is the derivative time constant, $\lambda$ and $\mu$ are the non-integer orders of the integral and derivative terms respectively.

The parameter $\mathrm{N}$ is chosen as $\mathrm{N}=10 \mathrm{~T}^{(\mu-1)}$. The performance index is integrated absolute error which is defined as follows

$$
M_{s}=\max \left\{\frac{1}{1+C(s) G(s)}\right\}
$$

Obviously, the higher value of $\mathrm{M}_{\mathrm{s}}$ yields the less robustness against uncertainties. If only the load disturbance rejection task is addressed, we have

$$
\begin{aligned}
K p & =\frac{1}{K}\left(a \exp \left(-b \frac{L}{T}\right)+c\left(\frac{L}{T}\right)^{d}\right) \\
T_{i} & =T^{\lambda}\left(a \exp \left(b \frac{L}{T}\right)+c \exp \left(d \frac{L}{T}\right)\right) \\
T_{d} & =\left(a\left(\frac{L}{T}\right)^{b}+c\right) T^{\mu}
\end{aligned}
$$

For the FOPID controllers, it is $\lambda=1$ 


$$
\mu=-0.04783\left(\frac{L}{T}\right)^{2}-0.01017 \frac{L}{T}+1.218
$$

for the series form, and

$$
\mu=0.06326\left(\frac{L}{T}\right)^{2}-0.1508 \frac{L}{T}+1.245
$$

for the ideal form.

\subsection{Examples:}

The transfer function

$$
G(s)=\frac{e^{-12.8 s}}{17.5 s+1}
$$

The normalized dead-time of the process is obtained as 0.4224 which implies that we can utilize the proposed tuning rules. The following FOPID can be obtained for the case of $\mathrm{M}_{\mathrm{s}}=1.4$ and $\mathrm{M}_{\mathrm{s}}=2.0$.

$$
\begin{gathered}
G_{C 1}(s)=0.5719\left(\frac{6.2220 s+1}{6.2220 s}\right)\left(\frac{9.9480 s^{1.2}+1}{0.5611 s^{1.2}+1}\right) \\
G_{C 2}(s)=0.3609\left(\frac{7.11 s+1}{7.11 s}\right)\left(\frac{9.7223 s^{1.1}+1}{0.731 s^{1.1}+1}\right)
\end{gathered}
$$

\section{Conclusion}

In this paper some of the well-known tuning methods of FOPID controllers are presented and several examples are illustrated, verifying the effectiveness of the methods are given. The optimization methods, Ziegler-Nichols tuning rules and the Padula\&Visioli methods were introduced. Based on these methods, we have considered the steam pressure model and the Vehicle wire control system. From these results, the potential of the fractional order controllers in practical industrial settings, regarding performance and robustness aspects, is clear. However, the design method proposed here involves complex equations relating the specifications of design and, sometimes, it may be difficult to find a solution to the problem. For this reason, the purpose now is to simplify the design method so that the controller can be tuned very easily, with very simple relations among its parameters, and preserving the robustness characteristics regarding performance, gain variations and noise.

\section{References}

[1] T. Hagglund and K. J. Astrom, "PID controllers: Theory, Design, and Tuning", ISA-The Instrumentation, Systems, and Automation Society (2nd edition), (1995).

[2] K. Kiong Tan, W. Qing-Guo, H. Chang Chieh and T. Hagglund, “Advances in PID Controllers”, Advances in Industrial Control, Springer- Verlag, London, (2000).

[3] S. P. Bhattacharyya Aniruddha Datta and M.-T. Ho, "Structure and Synthesis of PID Controllers", SpringerVerlag, London, (2000).

[4] I. Podlubny, "Fractional-order systems and $\mathrm{PI}^{\lambda} \mathrm{D}^{\mu}$-controllers," IEEE Trans. Automatic Control, vol. 44, (1999), pp. 208-214. 
[5] A. Oustaloup, F. Levron, B. Mathieu and F. M. Nanot, "Frequencyband complex noninteger differentiator: characterization and synthesis", IEEE Trans. Circuits Syst., I, vol. 47, (2000), pp. 25-39.

[6] C. A. Monje, B. M. Vinagre, Y. Q. Chen, V. Feliu, P. Lanusse and J. Sabatier, "Proposals for fraction PI ${ }^{\lambda} \mathrm{D}^{\mu}$ tuning", In: First IFAC Workshop on Fractional Differentiation and its Applications, Bordeaux, (2004).

[7] F. Padula and A. Visioli, "Tuning rules for optimal PID and fractional-order PID controllers", Journal of Process Control (2010), doi:10.1016/j.jprocont. 2010.10.006.

[8] F. Padula and A. Visioli, "Set-point Weighting for Fractional PID Controllers", Proceedings of Fractional Differentiation and its Applications, Badajoz, Spain, (2010) October.

[9] M. Tabatabaei and M. Haeri, "Loop Shaping Design of Fractional PD and PID Controllers", Proceedings of Fractional Differentiation and its Applications, Badajoz, Spain, (2010) October.

[10] A. Oustaloup, F. Levron, B. Mathieu and F. M. Nanot, "Frequencyband complex noninteger differentiator: characterization and synthesis," IEEE Trans., Circuits Syst. I, vol. 47, (2000), pp. 25-39.

[11] C. A. Monje, B. M. Vinagre, Y. Q. Chen, V. Feliu, P. Lanusse and J. Sabatier. "Proposals for fraction PI ${ }^{\lambda} \mathrm{D}^{\mu}$ tuning”, First IFAC Workshop on Fractional Differentiation and its Applications. Bordeaux, (2004).

\section{Authors}

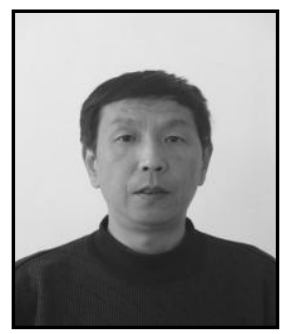

Zhe Yan is a professor at the department of automation from Harbin University of Science and Technology. His research area mainly includes electromagnetic compatibility and information process. E-mail: yanzhehrb@163.com 